\title{
ESTADO NUTRICIONAL, INDICADORES \\ ANTROPOMÉTRICOS Y HOMOCISTEÍNA SÉRICA EN MUJERES POSMENOPÁUSICAS VENEZOLANAS
}

\author{
NUTRITIONAL STATUS, ANTHROPOMETRIC \\ INDEX AND SERUM HOMOCYSTEINE IN \\ POSTMENOPAUSAL VENEZUELAN WOMEN
}

\author{
Lesbia Meertens (1), Nayka Díaz (1), Carlos Fraile (2), Melissa Riera (2), \\ Adelmo Rodríguez (2), Luis Rodríguez (2), Liseti Solano (1). \\ (1) Instituto de Investigaciones en Nutrición (INVESNUT). \\ Facultad de Ciencias de la Salud. Universidad de Carabobo. Valencia, Venezuela. \\ (2) Escuela de Medicina. Facultad de Ciencias de la Salud. \\ Universidad de Carabobo. Valencia, Venezuela.
}

\begin{abstract}
Menopause is associated with an increased body weight and changes in fat distribution, high levels of homocysteine and cardiovascular risk factors associated with estrogen deficiency. The objective was to evaluate body mass index (BMI), waist circumference (WC), waist/hip index (WHI), and serum homocysteine (Hct) levels in postmenopausal women (n 128). Nutritional status was diagnosed by BMI (WHO), WC (normal $<88 \mathrm{~cm}$, at risk $>88 \mathrm{~cm}$ ), WHI (normal $<0.8$, at risk $>0.8$ ), serum homocysteine $(\mathrm{Hct})(\mathrm{ELISA})$ normal $<10 \mathrm{mmol} / \mathrm{L}$, at risk $>10-15 \mathrm{mmol} / \mathrm{L}$, high $>15 \mathrm{mmol} / \mathrm{L}$ and estradiol (ELISA) $<65 \mathrm{pg} / \mathrm{mL}$ (menopause). Sixty five point nine \% were overweight/obese, $47.3 \%$ and $82.2 \%$ showed cardio metabolic risk by WC and WHI. There was a significant difference for WHI, and a positive significant correlation between anthropometrics indexes. Ten \% showed risk and hyperhomocysteinaemia, but it was not correlated with the evaluated variables. The subjects had a high frequency of overweight, obesity and android fat distribution, showing a high risk for cardiometabolics diseases.
\end{abstract}

Key words: Menopause, nutritional status, WC, WHI, homocysteine.

Este trabajo fue recibido el 9 de Julio de 2010 y aceptado para ser publicado el 20 de Junio de 2011.

\section{INTRODUCCIÓN}

La menopausia, etapa fisiológica de la vida de la mujer, se caracteriza por cambios significativos a nivel orgánico, psicológico y social, debido a modificaciones en la función endocrina, con disminución de los niveles circulantes de estrógenos, que dan lugar a alteraciones a nivel de la composición corporal, con el aumento del tejido adiposo y del peso corporal y modificaciones en la distribución de grasa, debido a la marcada influencia de estas hormonas sobre este tejido $(1,2)$.

Entre los indicadores antropométricos que evalúan estos cambios , se encuentran el índice de masa corporal (IMC), que permite categorizar el estado nutricional y diagnosticar estados de malnutrición por exceso como el sobrepeso y la obesidad, los cuales alcanzan frecuencias altas en las mujeres posmenopáusicas evaluadas (3).

La circunferencia de cintura (CCi), mide indirectamente la acumulación de grasa abdominal, y ha sido sugerida como un predictor de riesgo para las enfermedades cardiovasculares y metabólicas. Este indicador se ve afectado por los cambios en la distribución de la grasa corporal, observado en la posmenopausia (3-5).

La disminución de los estrógenos en la mujer posmenopáusica no sólo contribuye a la existencia de alteraciones a nivel de la composición corporal, sino también a la elevación de los valores de homocisteína. Este, es un amino ácido azufrado producido intercelularmente por la desmetilación de la metionina. Evidencias epide- 
miológicas, muestran que la homocisteína es un factor de riesgo independiente para la enfermedad vascular, que contribuye para la manifestación precoz de la enfermedad arterial coronaria periférica. La concentración plasmática de este aminoácido, es influenciada tanto por factores nutricionales, como el status del ácido fólico y las vitaminas $\mathrm{B}_{6} \mathrm{y} \mathrm{B}_{12}$, como por factores hereditarios, principalmente ligados a las enzimas del metabolismo de la metionina y cisteína, además de estados patológicos como la disminución del funcionalismo renal (6).

También el estilo de vida, puede modificar los niveles de este aminoácido, como hábitos tabáquicos, consumo de alcohol y de cafeína (7).

La hiperhomocisteinemia parece causar principalmente alteraciones del endotelio vascular, mediadas por el efecto tóxico-oxidativo del aminoácido. En el plasma, parte de éste es auto-oxidada, formando superóxidos y peróxidos de hidrógeno, lo que podría causar lesión de la célula endotelial, activación plaquetaria y trombosis (6).

Estudios epidemiológicos evidencian que la hiperhomocisteinemia, es más frecuente en mujeres posmenopáusicas, considerándose un factor de riesgo para enfermedades cardiovasculares, por lo que en esta etapa de la vida, las tasas de morbi-mortalidad por patologías cardiovasculares se incrementan considerablemente; sin embargo, otros estudios no lo reportan. Esta discordancia es atribuida a variaciones poblacionales, al tamaño muestral y métodos empleados en la determinación de la homocisteína (4-7).

El objetivo de este estudio fue asociar los niveles séricos de homocisteína con el estado nutricional antropométrico, factores de riesgos cardiometabólicos en mujeres posmenopáusicas.

\section{SUJETOS Y MÉTODOS}

La población de este estudio estuvo representada por 200 mujeres entre 45 y 60 años de edad, que asisten a consultas en los Centros de Salud dependientes de la Alcaldía del municipio Naguanagua, Estado Carabobo, región urbana, centro-norte de Venezuela.

La muestra seleccionada quedó constituida por 128 posmenopáusicas con criterios de inclusión de amenorrea de un año o más, sin terapia de reemplazo hormonal, aparentemente sanas, sin antecedentes de patologías cardiovasculares ni diabéticas. Los valores de estradiol sérico $(<65 \mathrm{pg} / \mathrm{mL})$, corroboraron el estado de posmenopausia.

La investigación se llevó a cabo siguiendo las Normas de Bioética para seres humanos (8), explicando los propósitos, beneficios y riesgos inherentes al estudio, aprobado por el Comité de Ética de la Facultad de Ciencias de la Salud, Universidad de Carabobo. Las participantes firmaron consentimiento escrito, previo al comienzo de la investigación. Este estudio formó parte de una valoración integral clínica del estado nutricional y de factores de riesgo cardiovascular, que incluyó determinaciones bioquímicas de glicemia, lípidos, vitaminas hidrosolubles y liposolubles y evaluación dietaria.

Se registraron los datos personales en una ficha diseñada para tal fin, incluyendo factores de estilo de vida tales como hábitos tabáquicos, consumo de alcohol y café, suplementos vitamínicos y uso de medicamentos. Se determinaron variables antropométricas y bioquímicas.

Evaluación antropométrica: Se realizó con ropa ligera y sin zapatos; se midió el peso en una balanza marca Detecto, calibrada; la talla con una cinta métrica no extensible de escala milimetrada, adosada a la pared y a $50 \mathrm{~cm}$ del piso, con una precisión de $100 \mathrm{~g}$ y $0,1 \mathrm{~cm}$ respectivamente, siguiendo las técnicas sugeridas por Gibson (9).

Con dichas mediciones, se calculó el índice de masa corporal (IMC) (peso/talla ${ }^{2}$ ) para realizar el diagnóstico del estado nutricional según OMS, considerándose déficit: $\leq 18,5 \mathrm{Kg} / \mathrm{m}^{2}$; normal: $18,5-24.9 \mathrm{Kg} / \mathrm{m}^{2}$; sobrepeso: $25-29,9 \mathrm{Kg} / \mathrm{m}^{2}$; obesidad: $\geq 30 \mathrm{Kg} / \mathrm{m}^{2}(10)$.

Se determinó la circunferencia de cintura $(\mathrm{CCi})$ y la de cadera $(\mathrm{CCa})$ con una cinta métrica no extensible (9). El resultado se expresó en centímetros. A partir de estos datos se calculó el índice cintura/cadera (ICC). Los valores de referencia para la $\mathrm{CCi}$ fueron normal: $\leq 88$ $\mathrm{cm}$; en riesgo: $>88 \mathrm{~cm}$ y para la ICC normal: $\leq 0,8$ y en riesgo $>0,8$, según criterios de ATP III (11).

Evaluación bioquímica: Las participantes del estudio acudieron en las primeras horas de la mañana, previo ayuno de 12 horas. Se extrajeron $3 \mathrm{cc}$ de sangre de la vena antecubital, que fueron colocados en tubo de polipropileno sin anticoagulante. Luego de centrifugado, el suero obtenido fue fraccionado en tubos, y almacenados a $-70 \mathrm{C}^{\mathrm{o}}$ hasta el momento del análisis de homocisteína y estradiol. La determinación de homocisteína sérica se realizó mediante ensayo enzimático (DIAZY$\mathrm{ME})$. Sus niveles fueron categorizados como: normal: $5-10 \mu \mathrm{mol} / \mathrm{L}$, riesgo: $10-15 \mu \mathrm{mol} / \mathrm{L}$, alto: $>15 \mu \mathrm{mol} / \mathrm{L}$.

Los niveles de estradiol sérico se determinaron mediante el método de inmunoensayo enzimático (DRG Diagnostics). Los valores de referencia según el kit utilizado fueron: posmenopausia: $0-65 \mathrm{pg} / \mathrm{mL}$.

La creatinina sérica se determinó por el método colorimétrico, para descartar trastornos de la función renal, siendo su valor de referencia: 0,8-1,2mg/dL (9).

Para el análisis de los datos se utilizó el programa SPSS versión 11.0. Se utilizó la prueba de KolmogorovSmirnov, para verificar la distribución de la normalidad de las variables en estudio (12). Se determinaron estadís- 
ticos descriptivos (media, desviación estándar); la comparación de medias se llevó a cabo mediante las pruebas no paramétricas de Mann-Whitney y Kruskal Wallis. Se usó $\mathrm{Chi}^{2}$ para las asociaciones de variables; el coeficiente de correlación de Spearman y probabilidad de riesgo. El intervalo de confianza fue de $95 \%$ (p:<0,05).

\section{RESULTADOS}

La tabla 1 muestra los valores promedios y rangos de las variables. El IMC para el grupo estuvo en la categoría de sobrepeso; sin embargo, se puede observar que hubo mujeres con obesidad mórbida. La CCi y el ICC en situación de riesgo.

Los niveles séricos de estradiol corroboraron el estado de posmenopausia de las mujeres evaluadas. La homocisteína y la creatinina sérica tuvieron valores promedios dentro del rango normal.

La tabla 2 evidencia que la malnutrición por exceso predominó en el grupo. No hubo diferencia significativa en relación a la clasificación del estado nutricional y CCi. En cuanto al ICC, la mayoría estuvo en situación de riesgo, mientras que predominaron los niveles normales de homocisteína sérica, siendo significativa la diferencia al comparar con los grupos en riesgo y alto.

En la tabla 3 se observan diferencias significativas en las variables antropométricas según las categorías del estado nutricional. No hubo mujeres en situación de déficit. Los niveles de estradiol fueron más elevados en el grupo con obesidad, con significancia estadística. La homocisteína sérica estuvo en el rango normal en todos los grupos.

El riesgo relativo de presentar una circunferencia de cintura en riesgo para enfermedades cardiovasculares fue de 0,82 , mientras que para el ICC, el riesgo fue de 1,42.

Se observó una correlación positiva y significativa entre niveles séricos de estradiol y variables antropométricas. La homocisteína sérica no correlacionó con las medidas evaluadas.

\section{DISCUSIÓN}

Durante el período de la menopausia se ha observado un incremento de los factores de riesgo de enfermedad cardiovascular en la mujer (13). Esta etapa favorece la ganancia de peso y el desarrollo de sobrepeso y obesidad. En la regulación del peso corporal intervienen numerosos mecanismos, entre ellos los hormonales. La declinación de los niveles de estrógenos que caracteriza la menopausia, se asocia al aumento de la grasa y peso corporal y a los cambios en la distribución del tejido adiposo que ocurren en este periodo $(14,15)$.

En este estudio, las mujeres presentaron un IMC promedio, indicativo de sobrepeso, predominando la malnutrición por exceso en el grupo. A la concentración de estradiol no se le puede atribuir por si sola el sobrepeso y la obesidad en esta muestra, ya que dado el carácter multifactorial de éstas condiciones, podrían estar influyendo otros factores como el estrato socioeconómico, los hábitos alimentarios inadecuados, la ausencia de actividad física y la edad, que favorecen el depósito graso y la sustitución de la masa magra por tejido adiposo (16-18), presentes en esta muestra. Estos resultados identifican un elevado número de estas mujeres en riesgo metabólico cardiovascular.

Hak y col (19), al evaluar mujeres posmenopáusicas, reportan un IMC en el límite inferior del rango de sobrepeso. Gavaler y col (20), muestran un IMC indicativo

\section{TABLA 1}

Caracterización de las variables antropométricas y bioquímicas en mujeres posmenopáusicas.

\begin{tabular}{lcc}
\hline \multicolumn{1}{c}{ Variables } & $\mathbf{X} \pm \mathbf{D E}$ & Rango \\
\hline Edad $(\mathrm{años})$ & $52,52 \pm 5,48$ & $45-60$ \\
$\mathrm{IMC}\left(\mathrm{kg} / \mathrm{m}^{2}\right)$ & $28,07 \pm 5,78$ & $18,5-46,5$ \\
Circunferencia cintura $(\mathrm{CCi})(\mathrm{cm})$ & $89,16 \pm 11,13$ & $67,6-124,0$ \\
Circunferencia cadera $(\mathrm{CCa})(\mathrm{cm})$ & $103,60 \pm 11,30$ & $83,0-143,0$ \\
Índice cintura cadera $(\mathrm{ICC})$ & $0,85 \pm 0,06$ & $0,69-1,08$ \\
Estradiol $(\mathrm{pg} / \mathrm{mL})$ & $28,38 \pm 18,11$ & $0,5-65$ \\
Homocisteína $(\mu \mathrm{mol} / \mathrm{L})$ & $7,89 \pm 3,10$ & $4,98-20,54$ \\
Creatinina $(\mathrm{mg} / \mathrm{dL})$ & $0,71 \pm 0,13$ & $0,30-1,10$ \\
\hline X \pm DE: promedio y desviación estándar. & & \\
\hline
\end{tabular}


de obesidad en mujeres posmenopáusicas hispánicas. Ambos autores atribuyen a factores hormonales y étnicos dichos hallazgos, ya que la declinación de los estrógenos circulantes predispone el aumento del peso corporal.

La menopausia favorece la distribución abdominal y visceral de la grasa, es decir, cambia el modelo ginecoide al androide. La CCi y el ICC se consideran indicadores sensibles para medir grasa abdominal (16). En la muestra evaluada, ambos indicadores están en situación de riesgo para patologías cardiometabólicas, observándose un elevado número de mujeres en riesgo según el ICC , hallazgos que pudieran explicarse por la edad y la declinación de la producción de estrógenos gonadales, que dan lugar a los cambios ya descritos en relación a la distribución de grasa corporal (15). Estos resultados coinciden con los reportados por Deibert y col, en mujeres posmenopáusicas (21) y a los de Piché y col (22), en un estudio realizado en mujeres posmenopáusicas donde reportaron una alta frecuencia de obesidad abdominal visceral, asociada a importantes factores de riesgo cardiovascular.

En este estudio, también se observó una correlación positiva entre estrógenos circulantes, IMC y CCi, pudiéndose inferir que la declinación de estrógenos predispuso a un mayor depósito de grasa y a la distribución de tipo androide. Moreno y col (17), también reportan una correlación positiva entre estos parámetros, al evaluar el estado nutricional de mujeres españolas de 35 a 55 años de edad, a diferencia de otros autores quienes manifiestan la independencia de estos dos indicadores en mujeres posmenopáusicas $(1,23)$.

En las mujeres con sobrepeso y obesidad se observó niveles más elevados de estradiol, pero dentro del rango para mujeres posmenopáusicas, dichos valores pudieran atribuirse a que el tejido adiposo sintetiza estrona a partir de la deshidroepiandrosterona, secretada por la corteza suprarrenal, por lo que la obesidad se asocia a una mayor producción endógena de estrógenos (24).

$\mathrm{La}$ ateroesclerosis es una enfermedad asociada a diversos factores de riesgo. Además, del aumento de

\section{TABLA 2}

Distribución de las mujeres posmenopáusicas según IMC, CCi, ICC y tHci sérica.

IMC

Normal

Sobrepeso

Obesidad

Total

$\mathrm{CCi}$

Normal

En riesgo

Total

ICC*

Normal

En riesgo

Total

tHci sérica**

Normal

En riesgo

Alto

Total

\section{n}

44

46

39

129

68

61

129

129

107

6

6

129
$\%$

34,1

35,7

30,2

100,0

52,7

47,3

100,0

17,8

82,2

100,0

100,0 
grasa corporal y su distribución, evidencias clínicas y epidemiológicas sugieren que una elevación de los niveles circulantes de homocisteína está asociada a la enfermedad vascular. El mecanismo de acción aceptado, es que el acúmulo de este aminoácido provoca daño a nivel endotelial con proliferación de células musculares y depósitos de lipoproteínas que afectan las plaquetas y el mecanismo de coagulación (25).

Algunos investigadores, han reportado un aumento de los niveles de homocisteína en la menopausia asociado a la alta incidencia de enfermedad vascular en esta etapa (26). En este estudio, los valores estuvieron dentro de los límites normales. Los niveles séricos de homocisteína parecen estar modulados por la edad, el tiempo de amenorrea y los niveles de estrógenos circulantes (26).

Estudios previos reportan que las concentraciones de este aminoácido aumentan $1 \mathrm{mmol} / \mathrm{L}$ por cada década, entre los 40 y los 70 años de edad, lo que se asocia a una disminución de las actividades de las enzimas que intervienen en el metabolismo de la homocisteína (27). En en este estudio, la edad promedio de las evaluadas se situó al inicio de la década de los 50 años, por lo que posiblemente este factor no influyó mayormente sobre la concentración de la homocisteína, tal como lo evidencian los niveles séricos obtenidos.

Hak y col (19), reportan una asociación entre concentración de homocisteína y tiempo de menopausia con una tendencia a elevarse después de cuatro años de amenorrea. En este estudio las mujeres tenían un promedio de 23 meses de posmenopausia.

Los niveles de estrógenos circulantes estimulan los procesos de transaminación de la metionina, lo que a su vez aumenta el catabolismo de la homocisteína. Morris y col (26) y Hak y col (19), reportaron niveles significativamente más altos de homocisteína en mujeres posmenopáusicas al comparar con premenopáusicas. La no coincidencia de estos resultados con los obtenidos en este estudio, podría explicarse por la alta prevalencia de sobrepeso y obesidad que había en las mujeres evaluadas, lo que sugiere un predominio del tejido adiposo sobre la masa muscular, que reflejaría niveles más bajos de homocisteína. Junto a esto, la producción de estrógenos endógenos a partir de la aromatización de andrógenos en el tejido adiposo, también podría explicar los niveles de homocisteina sérica más bajos encontrados en las mujeres obesas de la muestra (16).

Ganji y col (28), reportan una asociación negativa de homocisteína con IMC, en esta investigación la homocisteína no correlacionó con las variables antropométricas, similar a los resultados mostrados por Wolters y col (29).

Se concluye que el grupo evaluado presentó una alta frecuencia de sobrepeso/ obesidad y una distribución grasa de tipo androide. La homocisteína sérica estuvo dentro de los valores normales con una baja frecuencia de hiperhomocisteinemia.

Existe un alto riesgo de enfermedades cardiometabólicas que sugiere la necesidad de intervenir con

\section{TABLA 3}

\section{Variables antropométricas y bioquímicas en mujeres posmenopáusicas según estado nutricional por Índice de Masa Corporal (IMC)}

\section{Estado nutricional}

$\begin{array}{lcccc}\text { Variables } & \begin{array}{c}\text { Normal } \\ (\mathbf{n : ~ 4 4 )} \\ \mathbf{X} \pm \mathbf{D E}\end{array} & \begin{array}{c}\text { Sobrepeso } \\ (\mathbf{n : ~ 4 6 )} \\ \mathbf{X} \pm \mathbf{D E}\end{array} & \begin{array}{c}\text { Obesidad } \\ (\mathbf{n : ~ 3 9 )} \\ \mathbf{X} \pm \mathbf{D E}\end{array} & \mathbf{p} \\ \mathrm{IMC}\left(\mathrm{kg} / \mathrm{m}^{2}\right) & 22,7 \pm 1,6 & 27,0 \pm 1,4 & 35,1 \pm 4,6 & 0,000 \\ \mathrm{CCi}(\mathrm{cm} .) & 79,3 \pm 5,8 & 87,9 \pm 5,0 & 101,6 \pm 8,6 & 0,000 \\ \mathrm{CCa}(\mathrm{cm} .) & 94,8 \pm 5,6 & 101,0 \pm 4,4 & 116,5 \pm 10,2 & 0,000 \\ \text { ICC } & 0,8 \pm 0,05 & 0,8 \pm 0,05 & 0,8 \pm 0,07 & \\ \text { Estradiol }(\mathrm{pg} / \mathrm{mL}) & 25,0 \pm 19,2 & 26,9 \pm 17,5 & 4,8 \pm 17,0 & 0,024 \\ \text { Hct }(\mu \mathrm{mol} / \mathrm{L}) & 8,2 \pm 3,6 & 8,0 \pm 3,5 & 7,3 \pm 1,7 & \\ \text { Creatinina }(\mathrm{mg} / \mathrm{dL}) & 0,6 \pm 0,1 & 0,7 \pm 0,1 & 0,7 \pm 0,1 & \end{array}$

* Kruskal Wallis: p: $<0,05$

$\mathrm{X} \pm \mathrm{DE}$ : promedio y desviación estándar 
educación nutricional, para cambiar estilos de vida en relación a hábitos alimentarios y de actividad física en estas mujeres estudiadas.

\section{RESUMEN}

La menopausia se asocia a un aumento del peso corporal y a cambios en la distribución de grasa, describiéndose también niveles elevados de homocisteína, factores de riesgo cardiovascular asociados al déficit de estrógenos. El objetivo de este estudio fue evaluar índice de masa corporal (IMC), la circunferencia de cintura (CCi), el índice cintura-cadera (ICC), la homocisteína sérica en mujeres posmenopáusicas (n: 128). El diagnóstico nutricional antropométrico se determinó según IMC (OMS); se determinaron la CCi (normal $<88 \mathrm{~cm}$, en riesgo $>88 \mathrm{~cm}$ ), ICC (normal $<0,8$, en riesgo $>0,8)$, homocisteína sérica (tHci) (ELISA): normal < $10 \mathrm{mmol} / \mathrm{L}$, en riesgo $>10-15 \mathrm{mmol} / \mathrm{L}$, alta: $>15 \mathrm{mmol} / \mathrm{L}$ y estradiol (ELISA): $<65 \mathrm{pg} / \mathrm{mL}$ (posmenopausia). $65,9 \%$ presentaron sobrepeso/ obesidad. $47,3 \%$ y $82,2 \%$ en riesgo cardiometabólico según CCi e ICC. Hubo diferencia significativa para ICC y correlación positiva significativa entre indicadores antropométricos. $10 \%$ presentó riesgo e hiperhomocisteinemia. Este aminoácido no correlacionó con las variables evaluadas. Las mujeres evaluadas presentaron una alta frecuencia de sobrepeso-obesidad y una distribución de grasa tipo androide, presentando un alto riesgo para enfermedades cardiometabólicas.

Palabras clave: Menopausia, estado nutricional, CCi, ICC, homocisteína.

Dirigir la correspondencia a:

Profesora

Nayka Díaz

Instituto de Investigaciones en Nutrición

(INVESNUT).

Facultad de Ciencias de la Salud.

Universidad de Carabobo, Venezuela.

Celular: 58-416-6475925

Fax: 58-241-8672852

E-mail:ndiazw@gmail.com

Agradecimientos: Financiamiento del Consejo de Desarrollo Científico y Humanístico. Universidad de Carabobo (CDCH-UC).

\section{BIBLIOGRAFIA}

1. Garaulet M, Perez-Llamas F, Baraza J. Body fat distribution in pre-and post-menopausal women: metabolic and anthropometric variables. J Nutr Health Aging 2002; 6:123-26.

2. Mahabir S, Baer D, Johnson L, Roth M, Campbell
$\mathrm{W}$, Clevidence B et al. Body mass index, percent body fat and regional body fat distribution in relation to leptin concentrations in healthy, non-smoking postmenopausal women in a feeding study. Nutr J [serie en Internet] 2007 [citado 12 May 2009]; (6) 3: [cerca 7 p.]. Disponible en: http://www.nutritionj. com/content/6/1/3.pdf

3. Tardivo A, Nahas-Neto J, Nahas E, Maesta N, Rodrigues M, Orsatti F. associations between healthy eating patterns and indicators of metabolic risk in postmenopausal women. Nutr $\mathbf{J}$ [serie en Internet] 2010 [citado 26 May 2011]; (9)1: [cerca 9 p.]. Disponible en: http:// www.nutritionj.com/ content/9/1/64

4. Perry C, Alekel L, Ritland L, Bhupathiraju S, Stewart J, Hanson Let al. Centrally located body fat is related to inflammatory markers in healthy postmenopausal women.Menopause. [serie en Internet] 2008 [citado 26 Abr 2010]; 15: [cerca 8 p.]. Disponible en: http:// doi:10.1097/gme.0b013e318159f1a2

5. Koning L, Merchant A, Pogue J, Anand S. waist circumference and waist-to-hip-ratio as predictors of cardiovascular events: meta regression analysis of prospective studies. European Heart J [serie en Internet] 2007 [citado 26 May 2011]; 28: [cerca 6 p.]. Disponible en: http://doi:10.1093/eurheartj/ ehm026

6. Vannucchi H, Soares S. Hiper-homocisteinemia e risco cardiometabólico. Arq Bras Endocrinol Metab [serie en Internet] 2009 [citado 14 May 2010]; 53 : [cerca 9 p.]. Disponible en: http:// http://www. scielo.br/scielo.php?script=sci_arttext\&pid=S0004$27302009000500007 \& \operatorname{lng}=\mathrm{en} \& \mathrm{nrm}=\mathrm{iso}>$

7. Vollset SE, Refsum H, Tverdal A, Nygård O, Nordrehaug JE, Tell GS et al. Plasma total homocysteine and cardiovascular and noncardiovascular mortality: the Hordaland Homocysteine Study. Am J Clin Nutr 2001; 74 (1): 130-36.

8. Fonacit. gob.ve. [homepage on the Internet]. Caracas: Ministerio de Ciencia y Tecnología. Fondo Nacional de Ciencia, Tecnología e Innovación [actualizado 2002. [citado 18 Sept 20م8]. Disponible en: www.fonacit.gob.ve/bioetica.asp

9. Gibson R. Principles of Nutritional Assessment. Oxford University Press. New York. Oxford 1990.

10. WHO / EURO, OMS, PAHO / WHO. 2000. Disponible en: http://www.paho.org/Spanish/AD/DPC/ NC/cindi-diet.pdf.

11. Expert Panel on Detection. Evaluation and Treatment of High Blood Cholesterol in Adults. Executive Summary of the third report of the National Cholesterol Education Program (NCEP) Expert 
Panel on Detection, Evaluation and Treatment in High Blood Cholesterol in Adults. (Adult Treatment Panel III). JAMA. 2001; 285:2486-97.

12. Pardo A, Ruiz M. SPSS 11 Guía para el análisis de datos. Cap 11 Análisis exploratorio. El procedimiento explorar. México McGraw-Hill/Interamericana de España; 2002 p 205

13. Pérez L, Ramos L. Menopausia y Aterotrombosis. Rev Cubana Angiol y Cir Vasc 2002; 3(2):54-60.

14. Pavón de Paz I, Alameda H, Roldan O. Obesidad y Menopausia. Nutr Hosp 2006; 21: 633-7.

15. Milewicz A, Tworowska U, Demissie M. Menopausal obesity - myth of fact?. Climateric. 2001; 4:273-83.

16. Hadji P, Hars O, Bock K, Sturn G, Bauer T, Emons $\mathrm{G}$, Schultz K. The influence of menopause and body mass index on serum leptin concentrations. Eur $\mathbf{J}$ Endocrinol 2000; 143:55-60.

17. Moreno V, Gómez J, Gómez A, Antoranz M. Grasa corporal e índice adiposo-muscular estimados mediante Impedanciometría en la evaluación nutricional de mujeres de 35 a 55 años. Rev Esp Salud Pública. 2002; 76(6):723-34.

18. Lovejoy J. The Menopause and obesity. Prim Care Clin Office Pract. 2003; 30:317-25

19. Hak A, Polderman K, Westendorp I, Jakobs C, Hofman A, Witteman J. Increased plasma homocysteine after menopause. Atherosclerosis 2000; 149(1):163-8.

20. Gavaler J, Rosenblum E. Predictors of postmenopausal body mass index and waist ratio in the Oklahoma Postmenopausal Health Disparities Study. J Am Coll Nutr 2003; 22 (4):269-76.

21. Deibert P, König D, Vitolins MZ, Landmann U, Frey I, Zahradnik HP et al. Effect of a weight loss intervention on anthropometric measures and metabolic risk factors in pre-versus postmenopausal women. Nutrition J [serie en Internet] 2007 [citado 20 Abr 2009] 6 (31): [cerca 7 p.]. Disponible en: http:// nutritionj.com/content/6/1/31

22. Piché ME, Weisnagel S, Corneau L, Nadeau A, Bergeron J, Lemieux S. Contribution of abdominal visceral obesity and insulin resistance to the cardiovascular risks profile of potmenopausal women. Diabetes. [serie en Internet] 2005 [citado $20 \mathrm{Abr}$ 2009]. 54 (3): [cerca 7 p.]. Disponible en: http:// diabetes.diabetesjournals.org/content/54/3/770.pdf

23. Ozbey N, Sencer E, Molvalilar S, Orhan Y. Body fat distribution and cardiovascular disease risk factors in pre and postmenopausal obese women with similar BMI. Endocr J 2002; 49(4):503-9.

24. Ahn J, Schatzkin A, Lacey J, Albanes D, BallardBarbash R, Adams K et al. Adiposity, adult weight change, and postmenopausal breast cancer risk. Arch Intern Med [serie en Internet] 2007. [citado 30 May 2011] 167(19): [cerca 11 p.]. Disponible en: http:// archinte.ama-assn.org/cgi/reprint/167/19/2091

25. Dimitrova K, DeGroot K, Myers A, Kim Y. Estrogen and homocysteine. Cardiovasc Res. 2002; 53:577-88.

26. Morris M, Jacques P, Selhub J, Rosenberg I. Total homocysteine and estrogen status indicators in the Third National Health and Nutrition Examination Survey. Am J Epidemiol 2000; 152:140-8.

27. Holst-Schumacher I, Monge-Rojas R, CambroneroGutiérrez P, Brenes G. Genetic, dietary, and other lifestyle determinants of serum homocysteine levels in young adults in Costa Rica. Rev Panam Salud Pública. 2005; 17 (4):263-70.

28. Ganji V, Kafai M. Demographic, health, lifestyle, and blood vitamin determinants of serum total homocysteine concentrations in the Third National Health and Nutrition Examination Survey, 19881994. Am J Clin Nutr 2003; 77: 826-33.

29. Wolters M, Hermann S, Hahn A. B vitamin status and concentrations of homocysteine and methylmalonic acid in elderly German woman. Am J Clin Nutr 2003; 78: 765-72. 